\title{
OSCILLATION AND COMPARISON THEOREMS FOR CERTAIN NEUTRAL DIFFERENCE EQUATIONS
}

\author{
B. S. LALLI ${ }^{1}$ and B. G. ZHANG ${ }^{2}$ \\ (Received 1 November 1990; revised 3 November 1991)
}

\begin{abstract}
Some comparison theorems and oscillation criteria are established for the neutral difference equation

$$
\Delta\left(x_{n}+c x_{n-m}\right)+p_{n} x_{n-k}=0, \quad n=0,1,2, \ldots,
$$

as well as for certain neutral difference equations with coefficients of arbitrary sign. Neutral difference equations with mixed arguments are also considered.
\end{abstract}

\section{Introduction}

The problem of oscillation and nonoscillation of solutions of delay difference equations has received a great amount of attention in the last few years. Erbe and Zhang [1], Gedrgiou, Grove and Ladas [2], Ladas, Philos and Sficas [4], and Gyori and Ladas [3] have done extensive work on this topic. The problem of oscillation of all solutions of the neutral difference equation

$$
\Delta\left(y_{n}+c y_{n-m}\right)+p_{n} y_{n-k}=0, \quad n=0,1,2, \ldots,
$$

has been investigated in $[2,8]$, where $\Delta$ denotes the forward difference operator:

$$
\Delta y_{n}=y_{n+1}-y_{n} .
$$

The main purpose of this paper is to establish some oscillation and comparison results for neutral difference equations.

In Section 2 we obtain some comparison results for the oscillation of (1.1). To the best of our knowledge this is the first time that the comparison results

\footnotetext{
${ }^{1}$ Department of Mathematics, University of Saskatchewan, Saskatoon, Canada, S7N OWO.

${ }^{2}$ Dept. of Applied Math., China.

(C) Copyright Australian Mathematical Society 1992, Serial-fee code 0334-2700/92
} 
for (1.1) have been presented. An oscillation criterion for the neutral difference equations with positive and negative coefficients is given in Section 3. In Section 4 we consider the oscillation of some neutral difference equations with mixed arguments.

Let $M=\max \{m, k\}$, where $m$ and $k$ are non-negative integers. By a solution to (1.1) we mean a sequence $\left\{y_{n}\right\}$ which is defined for $n \geq N-M$ and which satisfies (1.1) for all $n \geq N$. Clearly if

$$
y_{n}=A_{n}, \quad \text { for } n=N-M, \ldots, N,
$$

are given, then (1.1) has a unique solution satisfying the initial conditions (1.2), where $N$ is an initial point. A nontrivial solution $\left\{y_{n}\right\}$ of (1.1) is said to be oscillatory if for every $N>0$ there exists an $n \geq N$ such that $y_{n} y_{n+1} \leq 0$. Otherwise it is called nonoscillatory.

Difference equations are appropriate models for describing situations where population growth is not continuous but seasonal, with overlapping generations. For example, the difference equation

$$
y_{n+1}=y_{n} \exp \left[r\left(1-\frac{y_{n}}{K}\right)\right]
$$

has been used to model various animal populations. It is also known [6] that for certain values of parameter $r$, the behavior of the solutions to (1.3) is chaotic. In fact for $r>3.102$ there are orbits of period three and this implies chaos. Equation (1.3) is considered by some to be the discrete analogue of the logistic differential equation

$$
y^{\prime}(t)=r y(t)\left[1-\frac{y(t)}{K}\right],
$$

where $r$ and $K$ are the growth rate and the carrying capacity of population respectively. One can study (1.3) by combining the linearisation method employed by Ladas [6] and the comparison methods developed in this paper. From the point of view of applications it is important to study neutral difference equations, since such equations are discrete analogues of neutral delay differential equations which appear in problems dealing with networks containing lossless transmission lines. Such networks arise in high speed computers where transmission lines are used to interconnect circuits.

\section{Main results}

First we establish a necessary and sufficient condition for the oscillation of (1.1). This condition turns out to be very useful for deriving sufficient conditions for oscillation of neutral difference equations with mixed arguments and equations with nonlinear terms. 
Our first result is the following:

TheOREM 2.1. Assume that $-1<c \leq 0, p_{n}>0$. Then every solution of (1.1) is oscillatory if and only if

$$
\Delta\left(y_{n}+c y_{n-m}\right)+p_{n} y_{n-k} \leq 0
$$

has no eventually positive solution.

Proof. The sufficiency is obvious. Suppose $\left\{y_{n}\right\}$ is an eventually positive solution of (2.1). We shall show that (1.1) has a positive solution also.

Let

$$
Z_{n}=y_{n}+c y_{n-m} \text {. }
$$

Then

$$
\Delta Z_{n}<0, \quad Z_{n}>0 \text { eventually. }
$$

Define

$$
w_{n}=-\frac{\Delta Z_{n}}{Z_{n}}>0, \quad n \geq N .
$$

It is obvious that $w_{n}<1$ for $n \geq N$. Inequality (2.1) can be rewritten in the form

$$
\Delta Z_{n}+p_{n} Z_{n-k}-c \frac{p_{n}}{p_{n-m}} p_{n-m} y_{n-m-k} \leq 0 .
$$

Dividing (2.4) by $Z_{n}$ and using (2.3) we have

$$
w_{n} \geq p_{n} \prod_{i=n-k}^{n-1}\left(1-w_{i}\right)^{-1}-c \frac{p_{n}}{p_{n-m}} w_{n-m} \prod_{i=n-m}^{n-1}\left(1-w_{i}\right)^{-1}
$$

Define

$$
\begin{gathered}
\left\{\lambda_{n}^{(0)}\right\}=\{0\}, \quad n=N, N+1, \ldots \quad \text { for } n=N, \ldots, N+M-1, \\
\left\{\lambda_{n}^{(r)}\right\}=\left\{p_{n} \prod_{j=n-k}^{n-1}\left(1-\lambda_{j}^{(r-1)}\right)^{-1}-c \frac{p_{n}}{p_{n-m}} \lambda_{n-m}^{(r-1)} \prod_{j=n-m}^{n-1}\left(1-\lambda_{j}^{(r-1)}\right)^{-1}\right\} \\
\text { for } n \geq N+M .
\end{gathered}
$$

It is not difficult to prove that

$$
\lambda_{n}^{(0)} \leq \lambda_{n}^{(1)} \leq \cdots \leq \lambda_{n}^{(r)} \leq \cdots \leq w_{n}, \quad r \geq 0, n \geq N .
$$

Then for each fixed $n \geq N$, we have

$$
\lim _{r \rightarrow \infty} \lambda_{n}^{(r)}=\lambda_{n}
$$


Taking the limit in (2.6), we get

$$
\begin{aligned}
& \lambda_{n}=p_{n} \prod_{j=n-k}^{n-1}\left(1-\lambda_{j}\right)^{-1}-c \frac{p_{n}}{p_{n-m}} \lambda_{n-m} \prod_{j=n-m}^{n-1}\left(1-\lambda_{j}\right)^{-1}, \quad n \geq N+M, \\
& \lambda_{n}=0, \quad n=N, \ldots, N+M-1 .
\end{aligned}
$$

Define

$$
\begin{aligned}
Z_{N} & =1 \\
Z_{n+1} & =Z_{n}\left(1-\lambda_{n}\right) \quad \text { for } n \geq N .
\end{aligned}
$$

That is,

$$
\begin{aligned}
Z_{N+1} & =\left(1-\lambda_{N}\right) \\
Z_{N+2} & =\left(1-\lambda_{N}\right)\left(1-\lambda_{N+1}\right) \\
\cdots & =\cdots \\
Z_{n} & =\prod_{i=N}^{n-1}\left(1-\lambda_{i}\right)>0 \quad \text { for } n \geq N .
\end{aligned}
$$

Thus

$$
\Delta Z_{n}=-Z_{n} \lambda_{n}<0
$$

So

$$
\lambda_{n}=-\frac{\Delta Z_{n}}{Z_{n}}>0
$$

Substituting (2.10) into (2.8), we have

$$
-\frac{\Delta Z_{n}}{Z_{n}}=p_{n} \frac{Z_{n-k}}{Z_{n}}-c \frac{p_{n}}{p_{n-m}}\left(l-\frac{Z_{n-m+1}}{Z_{n-m}}\right) \frac{Z_{n-m}}{Z_{n}}, \text { for } n \geq N+M .
$$

So

$$
-\Delta Z_{n}=p_{n} Z_{n-k}-c \frac{p_{n}}{p_{n-m}}\left(-\Delta Z_{n-m}\right), \quad \text { for } n \geq N+M
$$

or

$$
-\frac{\Delta Z_{n}}{p_{n}}=Z_{n-k}+\frac{c}{p_{n-m}} \Delta Z_{n-m}, \quad \text { for } n \geq N+M
$$

Define

$$
x_{n}=-\frac{\Delta Z_{n+k}}{p_{n+k}}>0, \quad \text { for } n \geq N+M-k .
$$

By combining (2.11) and (2.12), we obtain

$$
x_{n-k}=Z_{n-k}-c x_{n-m-k}, \text { for } n \geq N+M,
$$


that is

$$
Z_{n-k}=x_{n-k}+c x_{n-k-m} .
$$

Now substituting (2.13) into (2.12), we get

$$
\Delta\left(x_{n}+c x_{n-m}\right)+p_{n} x_{n-k}=0 \text {, for } n \geq N+M \text {. }
$$

Thus, $\left\{x_{n}\right\}, n \geq N+M-k$, is a positive solution of (1.1), which is a contradiction. This completes the proof of the theorem.

REMARK 2.1. Theorem 1 in [9] is a special case of Theorem 2.1.

The following results illustrate applications of Theorem 2.1. We consider

$$
\Delta\left(y_{n}+c y_{n-m}\right)+p_{n} y_{n-k}+f\left(n, y_{n-h_{1}}, \ldots, y_{n-h_{l}}\right)=0
$$

and establish the following:

THEOREM 2.2. Suppose that the assumptions of Theorem 2.1 hold and that

$$
f\left(n, \xi_{1}, \ldots, \xi_{l}\right) \xi_{1} \geq 0 \text { whenever } \xi_{1} \xi_{j}>0, j=1, \ldots, l .
$$

Then the oscillation of (1.1) implies the oscillation of (2.14).

Proof. If not, without loss of generality let $\left\{y_{n}\right\}$ be an eventually positive solution of (2.14). Then

$$
\Delta\left(y_{n}+c y_{n-m}\right)+p_{n} y_{n-k} \leq 0,
$$

has an eventually positive solution which contradicts the conclusion of Theorem 2.1.

REMARK 2.2. It follows that the oscillation of (1.1) implies that of

$$
\Delta\left(y_{n}+c y_{n-m}\right)+p_{n} y_{n-k}+q_{n} y_{n+h}=0
$$

where

$$
-1<c \leq 0, p_{n} \geq 0, q_{n} \geq 0, k, h>0 .
$$

Next we consider the equation

$$
\Delta\left(y_{n}+\bar{c} y_{n-m}\right)+q_{n} y_{n-k}=0,
$$

and derive the following result:

TheORem 2.3. Assume that $\bar{c}, c \in(-1,0]$ and that

$$
\begin{gathered}
q_{n} \geq p_{n}>0, \\
\bar{c} q_{n} / q_{n-m} \leq c p_{n} / p_{n-m} .
\end{gathered}
$$


Then the oscillation of (1.1) implies that of (2.16).

Proof. If not, let $\left\{y_{n}\right\}$ be a positive solution of (2.16), for $n \geq N$. Then as in the proof of Theorem 2.1, there exists a sequence $\left\{w_{n}\right\}, w_{n} \in(0,1)$ such that

$$
w_{n}=q_{n} \prod_{i=n-k}^{n-1}\left(1-w_{i}\right)^{-1}-\bar{c} \frac{q_{n}}{q_{n-m}} w_{n-m} \prod_{i=n-m}^{n-1}\left(1-w_{i}\right)^{-1} .
$$

In view of (2.17) and (2.18) it follows from (2.19) that

$$
w_{n} \geq p_{n} \prod_{i=n-k}^{n-1}\left(1-w_{i}\right)^{-1}-c \frac{p_{n}}{p_{n-m}} w_{n-m} \prod_{i=n-m}^{n-1}\left(1-w_{i}\right)^{-1}
$$

Thus we get the inequality (2.5), which leads to the conclusion that (1.1) has a positive solution, and hence a contradiction.

THEOREM 2.4. If

$$
\begin{gathered}
q_{i} \geq p_{i}>0, \\
\sum_{i=N}^{\infty} q_{i}=\infty, \quad-1<\bar{c} \leq c<0,
\end{gathered}
$$

then the oscillation of (1.1) implies the oscillation of (2.16).

Proof. Suppose the contrary, and let $\left\{y_{n}\right\}$ be a positive solution of (2.16). With

$$
Z_{n}=y_{n}+\bar{c} y_{n-m}
$$

we have

$$
\Delta Z_{n}+q_{n} y_{n-k}=0 \text {. }
$$

It is easy to verify (see [8]) that $Z_{n} \rightarrow 0$ as $n \rightarrow \infty$.

Therefore

$$
Z_{n}=\sum_{n}^{\infty} q_{i} y_{i-k}
$$

So

$$
y_{n}=-\bar{c} y_{n-m}+\sum_{n}^{\infty} q_{i} y_{i-k} \geq-c y_{n-m}+\sum_{n}^{\infty} p_{i} y_{i-k},
$$

and hence

$$
\begin{gathered}
y_{n} \geq-c y_{n-m} \geq \cdots \geq(-c)^{l} y_{n-l m}=(-c)^{\left(n-n_{0}\right) / m} y_{n_{0}}=\alpha(-c)^{n / m}, \\
\alpha=(-c)^{-n_{0} / m} y_{n_{0}} .
\end{gathered}
$$




\section{Define}

$$
\left.\begin{array}{ll}
\left\{\lambda_{n}^{(0)}\right\}=\left\{y_{n}\right\}, & n \geq n_{0} \\
\lambda_{n}^{(r)}=-c \lambda_{n-m}^{(r-1)}+\sum_{n}^{\infty} p_{i} \lambda_{i-k}^{(r-1)}, & n \geq n_{0}+\max \{m, k\} \\
\lambda_{n}^{(r)}=\lambda_{n}^{(0)}, \text { for } n_{0} \leq n<n_{0}+\max \{m, k\}, & r=1,2,3, \ldots
\end{array}\right\} .
$$

It is obvious that

$$
\lambda_{n}^{(1)} \leq \lambda_{n}^{(0)}, \quad \text { for } n \geq n_{0}
$$

By induction

$$
\lambda_{n}^{(r+1)} \leq \lambda_{n}^{(r)}, \quad \text { for } r=0,1,2, \ldots, n \geq n_{0}
$$

and

$$
\lambda_{n}^{(r)} \geq \alpha(-c)^{n / m}, \quad \text { for } r=0,1,2, \ldots .
$$

Therefore for each $n \geq n_{0}$ we have

$$
\lambda_{n}^{(r)} \rightarrow \lambda_{n}^{*} \quad \text { as } r \rightarrow \infty
$$

From (2.23), one gets

$$
\lambda_{n}^{(*)}=-c \lambda_{n-m}^{*}+\sum_{i=n}^{\infty} p_{i} \lambda_{i-k}^{*}
$$

and hence

$$
\Delta\left(\lambda_{n}^{*}+c \lambda_{n-m}^{*}\right)=-p_{n} \lambda_{n-k}^{*}, \quad n \geq n_{0}+M .
$$

That is, $\left\{\lambda_{n}^{*}\right\}$ is a positive solution of (1.1), which is a contradiction.

COROLlaRY 2.1. If $c=\bar{c}=0$, then for the equations

$$
\Delta y_{n}+p_{n} y_{n-k}=0
$$

and

$$
\Delta x_{n}+q_{n} x_{n-k}=0,
$$

with $q_{n} \geq p_{n}, l \geq K$, the oscillation of (2.25) implies the oscillation of (2.26).

Proof. Follows from that of Theorem 2.3.

REMARK 2.3. This corollary was established by Yan and Chuanxi [9] recently.

REMARK 2.4. It is easy to extend the above results to

$$
\Delta\left(y_{n}+c y_{n-m}\right)+\sum_{i=1}^{l} p_{i n} y_{n-k_{i}}=0 \text {. }
$$


3. Neutral difference equations with positive and negative coefficients

We consider now neutral difference equations with positive and negative coefficients, of the form

$$
\Delta\left(y_{n}+c y_{n-m}\right)+p_{n} y_{n-k_{1}}-q_{n} y_{n-k_{2}}=0,
$$

and prove the following:

THEOREM 3.1. Assume that

$$
\begin{gathered}
-1<c \leq 0, p_{n} \geq 0, q_{n} \geq 0, k_{1}>k_{2}+1, \text { and } p_{n}-q_{n-\left(k_{1}-k_{2}\right)} \geq 0(\not \equiv 0), \\
\lim _{n \rightarrow \infty} \sum_{n-\left(k_{1}-k_{2}\right)}^{n-1} q_{i}=0
\end{gathered}
$$

Further assume that every solution of

$$
\Delta Z_{n}+\left(p_{n}-q_{n-\left(k_{1}-k_{2}\right)}\right) Z_{n-k_{1}}=0
$$

is oscillatory. Then every solution of (3.1) is oscillatory.

Proof. If not, let $\left\{y_{n}\right\}$ be a positive solution of (3.1). Define

$$
\begin{gathered}
Z_{n}=y_{n}+c y_{n-m} \\
w_{n}=Z_{n}-\sum_{n-\left(k_{1}-k_{2}\right)}^{n-1} q_{i} y_{i-k_{2}} .
\end{gathered}
$$

Then

$$
\begin{aligned}
\Delta w_{n} & =\Delta Z_{n}-q_{n} y_{n-k_{2}}+q_{n-\left(k_{1}-k_{2}\right)} y_{n-k_{1}} \\
& =\left(q_{n-\left(k_{1}-k_{2}\right)}-p_{n}\right) y_{n-k_{1}}
\end{aligned}
$$

Since $q_{n-\left(k_{1}-k_{2}\right)}-p_{n} \leq 0$ we have $\Delta w_{n} \leq 0$. If $w_{n} \rightarrow-\infty$ as $n \rightarrow \infty$, then $y_{n}$ must be unbounded. This implies that there exists an integer $N$ such that $w_{N}<0$,

$$
y_{N}=\max _{n \leq N}\left\{y_{n}>0\right\} \quad \text { and } \quad 1-c-\sum_{N-\left(k_{1}-k_{2}\right)}^{N-1} q_{i} \geq 0 .
$$


On the other hand,

$$
\begin{aligned}
0>w_{N} & =Z_{N}-\sum_{N-\left(k_{1}-k_{2}\right)}^{N-1} q_{i} y_{i-k_{2}} \\
& =y_{N}+c y_{N-M}+\sum_{N-\left(k_{1}-k_{2}\right)}^{N-1} q_{i} y_{i-k_{2}} \\
& \geq y_{N}\left(1+c-\sum_{N-\left(k_{1}-k_{2}\right)}^{N-1} q_{i}\right) \geq 0,
\end{aligned}
$$

which is impossible.

Thus we must have $\lim _{n \rightarrow \infty} w_{n}=l$, where $l$ is finite. Thus $y_{n}$ is bounded and from (3.4) we have $\lim _{n \rightarrow \infty} Z_{n}=l$. If $l<0$, then for $n$ sufficiently large

$$
y_{n}+c y_{n-m} \leq \frac{l}{2}<0 \text {. }
$$

It follows that $y_{n} \rightarrow 0$ and hence $Z_{n} \rightarrow 0$ as $n \rightarrow \infty$. Consequently $l$ cannot be negative. Therefore $l \geq 0$, and since $\Delta w_{n} \leq 0$, we have

$$
w_{n}=Z_{n}-\sum_{n-\left(k_{1}-k_{2}\right)}^{n-1} q_{i} y_{i-k_{2}} \geq 0 .
$$

That is, $y_{n} \geq Z_{n} \geq w_{n}$. Substituting this into (3.5) we have

$$
\Delta w_{n}+\left(p_{n}-q_{n-\left(k_{1}-k_{2}\right)}\right) w_{n-k_{1}} \leq 0 \text {. }
$$

Since

$$
p_{n}-q_{n-\left(k_{1}-k_{2}\right)} \not \equiv 0, \quad \Delta w_{n} \not \equiv 0,
$$

it follows that $w_{n}>0$ eventually, which implies that (3.3) has a positive solution. This contradicts the assumption that (3.3) is oscillatory. Thus the proof of the theorem is complete.

REMARK 3.1. It is known (see [1]) that every solution of (3.3) is oscillatory if

$$
\lim _{n \rightarrow \infty} \inf \left(p_{n}-q_{n-\left(k_{1}-k_{2}\right)}\right)>\frac{k_{1}^{k_{1}}}{\left(1+k_{1}\right)^{1+k_{1}}} .
$$

EXAmple 3.1. Consider

$$
\Delta y_{n}+p_{n} y_{n-4}-q_{n} y_{n-1}=0, \quad n>4,
$$

where

$$
p_{n}=\frac{2(n-4)}{n+1}, \quad q_{n}=\frac{n-1}{n(n+1)} .
$$


Equation (3.6) satisfies all the assumptions of Theorem 3.1. Therefore every solution of (3.6) is oscillatory. In fact $\left\{y_{n}\right\}=\left\{(-1)^{n} \frac{1}{n}\right\}$ is such a solution.

\section{Difference equations of mixed type}

For the following difference equation of the mixed type:

$$
\Delta\left(y_{n}+c y_{n-m}\right)+\sum_{i=1}^{l} p_{i} y_{n-k_{i}}=0,
$$

we have the following:

THEOREM 4.1. Assume that

(i) $-1<c<0$,

(ii) $p_{i}>0$ are constants, $m, k_{i}$ are integers, $m>0, k_{i}$ with arbitrary sign,

$$
\sum_{i=1}^{l} p_{i}(-c)^{\sigma_{i}}\left(\frac{\bar{k}_{i}+1}{\overline{k_{i}}}\right)^{\overline{k_{i}}}\left(\overline{k_{i}}+1+\frac{c m}{1+c}\right)>1+c
$$

where $\sigma_{i}$ is the smallest nonnegative integer such that

$$
\overline{k_{i}}=\sigma_{i} m+k_{i}>0, \quad i=1,2, \ldots, l .
$$

Then every solution of $(4.1)$ is oscillatory.

Proof. If not, let $\left\{y_{n}\right\}$ be a positive solution of (4.1). Put

$$
Z_{n}=y_{n}+c y_{n-m} \text {. }
$$

It is easy to see that there exists a sufficiently large integer $n_{0}$ such that

$$
Z_{n}>0, \Delta Z_{n}<0, \quad \text { for } n \geq n_{0} .
$$

From (4.3), we have

$$
\begin{aligned}
y_{n} & =\sum_{j=0}^{h}(-c)^{j} Z_{n-j m}+(-c)^{h+1} y_{n-(h+1) m} \\
& >\sum_{j=0}^{h}(-c)^{j} Z_{n-j m}, n \geq n_{0}+(h+1) m \\
y_{n-k_{i}} & >\sum_{j=0}^{h}(-c)^{j} Z_{n-k_{i}-j m}, \quad n \geq n_{0}+(h+1) m+\tau,
\end{aligned}
$$

where $\tau=\max _{1 \leq i \leq l}\left\{m,\left|k_{i}\right|\right\}$. 
Substituting (4.4) into (4.1) we get

$$
\Delta z_{n}+\sum_{i=1}^{l} p_{i}(-c)^{\sigma_{i}} \sum_{j=0}^{h-\sigma_{i}}(-c)^{j} Z_{n-\overline{k_{i}}-j m} \leq 0
$$

By Theorem 2.1, (4.5) implies that

$$
\Delta Z_{n}+\sum_{i=1}^{l} p_{i}(-c)^{\sigma_{i}} \sum_{j=0}^{h-\sigma_{i}}(-c)^{j} Z_{n-\bar{k}_{i}-j m}=0
$$

has a positive solution. On the other hand, from (4.2),

$$
\sum_{i=1}^{l} p_{i}(-c)^{\sigma_{i}}\left(\frac{\overline{k_{i}}+1}{\overline{k_{i}}}\right)^{\overline{k_{i}}}\left(\overline{k_{i}}+1-\frac{c m}{1+c}\right)>1+c,
$$

or

$$
\sum_{i=1}^{l} p_{i}(-c)^{\sigma_{i}}\left(\frac{\overline{k_{1}}+1}{\overline{k_{i}}}\right)^{\overline{k_{i}}}\left[\left(\overline{k_{i}}+1\right) \sum_{j=0}^{\infty}(-c)^{j}-c m \sum_{j=0}^{\infty}(j+1)(-c)^{j}\right]>1,
$$

which we have obtained by dividing both sides by $1+c$, and by using the fact that

$$
\left(\sum_{j=0}^{\infty} r^{j}\right)\left(\sum_{j=0}^{\infty} r^{j}\right)=\sum_{j=0}^{\infty}(j+1) r^{j}, \quad \text { for } 0<r<1 .
$$

Now we can choose $h>\sigma_{i}$ so that

$$
\begin{gathered}
\sum_{i=1}^{l} p_{i}(-c)^{\sigma_{i}}\left(\frac{\overline{k_{i}}+1}{\overline{k_{i}}}\right)^{\overline{k_{i}}}\left[\left(\overline{k_{i}}+1\right) \sum_{j=0}^{h-\sigma_{i}}(-c)^{j}-c m \sum_{j=0}^{h-\sigma_{i}-1}(j+1)(-c)^{j}\right]>1 \\
\text { i.e. } \sum_{i=1}^{l} p_{i}(-c)^{j}\left(\frac{\overline{k_{i}}+1}{\overline{k_{i}}}\right)^{\overline{k_{i}}}\left[\sum_{j=0}^{h-\sigma_{i}}(-c)^{j}\left(\overline{k_{i}}+1+j m\right)\right]>1 .
\end{gathered}
$$

Since $\left(\frac{x+1}{x}\right)^{x}$ is an increasing function for $x>0$, we have

$$
\left(\frac{\overline{k_{i}}+1}{\bar{k}_{i}}\right)^{\overline{k_{i}}}<\left(\frac{\overline{k_{i}}+1+j m}{\bar{k}_{i}+j m}\right)^{\overline{k_{i}+j m}}, \quad j>0 .
$$

We substitute (4.8) into (4.7) to obtain

$$
\sum_{i=1}^{l} p_{i}(-c)^{\sigma_{i}} \sum_{j=0}^{h-\sigma_{i}}(-c)^{j} \frac{\left(\overline{k_{i}}+1+j m\right)^{\overline{k_{i}}+1+j m}}{\left(\overline{k_{i}}+j m\right)^{\overline{k_{i}}+j m}}>1 .
$$


By a known result [1], (4.9) implies that every solution of (4.6) is oscillatory. This contradiction proves the theorem.

REMARK 4.1. If $k_{i}>0$, then $\sigma_{i}=0, \bar{k}_{i}=k_{i}$, and then (4.2) reduces to

$$
\sum_{i=1}^{l} p_{i}\left(\frac{k_{i}+1}{k_{i}}\right)^{k_{i}}\left(k_{i}+1+\frac{c m}{1+c}\right)>1+c .
$$

If we further assume that $c=0$, then (4.10) becomes

$$
\sum_{i=1}^{l} p_{i} \frac{\left(k_{i}+1\right)^{k_{i}+1}}{k_{i}^{k_{i}}}>1,
$$

which coincides with the result in [1].

\section{Acknowledgement}

Research of B. S. Lalli was partially supported by NSERC grant A5293, and partially by a grant from the President's fund.

\section{References}

[1] L. H. Erbe and B. G. Zhang, "Oscillation of discrete analogues of delay equation", Diff. Int. Equations 2 (1989) 300-309.

[2] D. A. Gedrgiou, E. A. Grove and G. Ladas, "Oscillations of neutral difference equations", Appl. Anal., 33, ND 3-4 (1989) 243-253.

[3] I. Gyori and G. Ladas, "Linearized oscillation for equations with piecewise constant arguments", Diff. Int. Equations 2 (1989) 123-131.

[4] G. Ladas, C. G. Philos and Y. G. Sficas, "Sharp conditions for the oscillation of delay difference equations", J. Applied Math and Simulation 2 (1989) 101-112.

[5] G. Ladas, C. G. Philos and Y. G. Sficas, "Necessary and sufficient conditions for the oscillation of difference equations", Liberta Math. 9 (1989) 121-125.

[6] G. Ladas, "Recent developments in the oscillation of delay equations", in Differential equations: Stability and Control, (Marcel Decker, 1990).

[7] G. Ladas, "Oscillations of difference equations with positive and negative coefficients", Conference on Differential equations and Population Biology, June 10-25, 1988, Edmonton, Canada.

[8] B. S. Lalli, B. G. Zhang and Li Juan Zhao, "On oscillations and existence of positive solutions of neutral difference equations", J. Math. Anal. Appl. 158 (1991) 213-233.

[9] Jurang Yuan and Chuanxi Qian, "Oscillation and comparison results for delay difference equations", J. Math. Anal. Appl. (to appear). 